\title{
My burning issues in TKI therapy and treatment-free remission in CML
}

\author{
Dominik Wolf
}

Received: 12 November 2017 / Accepted: 5 December 2017 / Published online: 21 December 2017

C Springer-Verlag GmbH Austria, part of Springer Nature 2017

We all agree that treatment-free remission (TFR) is a common goal we aim for in cancer treatment. Even though many malignant diseases require longterm/maintenance therapies (i. e., IMiD maintenance in myeloma, antihormonal therapy in hormone-receptor-positive breast cancer or JAK inhibitors in myelofibrosis patients ineligible for allo-SCT), this often induces clinically relevant side effects lowering quality of life (QoL) and frequently also requires more intense clinical monitoring. For years, we also communicated the necessity of continuous life-long tyrosine kinase inhibitor (TKI) therapy to our patients with chronic myeloid leukemia (CML), until seminal work by F.X. Mahon and his French colleagues demonstrated that even though in some patients lowlevel disease burden fluctuates upon TKI withdrawal, approximately half of those patients can be successfully discontinued from targeted therapy for the longterm [1]. However, only a small proportion of optimal responders can continuously stop therapy (overall a maximum of $25 \%$ of chronic phase CML [CP-CML] patients treated up-front with 2nd generation TKIs are in long-term TFR). However, for me the following questions remain to be answered.

How can we bring more patients into the appropriate corridor of deep molecular response to discontinue TKI therapy?

Even though 2nd generation TKIs are more potent in terms of deep molecular remission (DMR) compared to imatinib [2-4], still only approximately half of the

\section{Wolf, MD (凶)}

Medical Clinic III (Oncology, Hematology and

Rheumatology), University Clinic Bonn (UKB),

Sigmund-Freud-Straße 25, 53127 Bonn, Germany

dominik.wolf@ukb.uni-bonn.de
CP-CML patients qualify for a TKI discontinuation attempt [5]. Novel combination therapies of available TKIs together with novel drugs, such as ABL001 in patients with a lower likelihood to achieve continuous DMR should be tested [6]. Biomarkers such as high sCD62L [7] or high-risk scores (ETLS) [8] may help to select appropriate patients to test such drug combinations that also increase the risk of adverse events.

Depth of response and TKI therapy duration: how deep is deep and how long is long enough?

The required response depth remains unclear, as interestingly studies with varying selection criteria for patients to be discontinued almost all showed the same result of approximately $40-50 \%$ successful discontinuation rates. This already points to other predictive (e.g., immunological?) variables being mainly responsible to long-term TFR. However, it is generally accepted that continuous (at least 2 years) of molecular response 4 (MR4) during 2nd generation TKI therapy and 5.7 years with imatinib may be required to allow TKI discontinuation [9].

Which biomarkers predict successful treatmentfree remission?

Immunological biomarkers at the time of TKI discontinuation are linked to the likelihood of continuous TFR, including increased natural killer (NK) cells [10, 11] and CD86-positive plasmacytoid dendritic cells (pDC) [12] numbers and function, the latter being also linked to a $\mathrm{T}$ cell exhaustion phenotype with high PD1 expression potentially also at least in part explaining limited anti-leukemic potency of leukemia antigen(LAA)-specific cytotoxic T lymphocytes. The latter study also suggested that the number of CD86-positive $\mathrm{pDC}$ overrules the time effect of previous therapy 
on TFR likelihood, as in patients with high CD86 pDC numbers $(>95 / \mu \mathrm{l})$ the effect of previous TKI therapy duration does not at all impact TFR rates, whereas in patients with low CD86 pDC numbers, long-term TKI pretreated patients have a $90 \%$ success rate of being continuously TKI free.

How can we consolidate patients in DMR to improve the likelihood of TFR?

Novel study generations that randomize nonconsolidation versus consolidation therapy upon TKI stop have to be developed. The German CML study group will soon start two prospective clinical trials under the lead of Professor Burchert from Marburg validating innovative concepts of immunoconsolidation in patients with DMR but nonselected for the above mentioned immunological variables. The first strategy validates low-dose maintenance therapy with the novel ropeginterferon [13] as compared to stopping without consolidation. The second concept is based on the observation described above that numbers of CD86expressing pDC correlate with $\mathrm{PD}-1$ expressing proteinase-3 (i. e., leukemia antigen=LAA) specific CD8 $\mathrm{T}$ cells. Moreover, CD86 is the ligand for CTLA- 4 that is expressed on regulatory $\mathrm{T}$ cells and which may be involved in $\mathrm{T}$ cell dysregulation in CD86+ pDC high patients. Thus, a combined immune-activating consolidation therapy of the CTLA-4 blocking monoclonal antibody ipilimumab together with the PD-1 blocker nivolumab will be tested against a nonintervention approach in patients with CML in DMR and TKI discontinuation.

The studies exemplify how future consolidation approaches may help to make use of an improved immune-surveillance upon TKI discontinuation to improve TFR rates.

\section{Conclusion and open questions}

Maintenance concepts are interesting but may also induce relevant side effects. Consolidation strategies are only of added value when they are indeed able to either reduce residual leukemic burden or induce immunosurveillance leading to an increased TFR rate or median duration. Immunotherapy concepts also may induce side effects, such as immune-related adverse events, which have to be closely monitored. Treatment in well-controlled clinical trials is thus warranted to define innovative strategies with the ultimate goal to induce improved long-term TFR rates without relevant side effects. This will profoundly impact QoL for treatment-free patients as well as it will also be of substantial pharmacoeconomic benefit.

Conflict of interest D. Wolf declares that he has no competing interests.

\section{References}

1. Etienne G, GuilhotJ, Rea D, et al. Long-term follow-up of the French stop Imatinib (STIM1) study in patients with chronic myeloidleukemia. JClin Oncol. 2017;35:298-305.

2. Cortes JE, Gambacorti-Passerini C, Deininger MW, et al. Bosutinib versus Imatinib for newly diagnosed chronic myeloid leukemia: results from the randomized BFORE trial. J Clin Oncol. 2017; https:// doi.org/10.1200/JCO.2017. 74.7162.

3. Hochhaus A, Saglio G, Hughes TP, et al. Long-term benefits and risks of frontline nilotinib vs imatinib for chronic myeloid leukemia in chronic phase: 5 -year update of the randomizedENESTnd trial. Leukemia. 2016;30:1044-54.

4. Jabbour E, Kantarjian HM, Saglio G, et al. Early response with dasatinib or imatinib in chronic myeloid leukemia: 3 -year follow-up from a randomized phase 3 trial (DASISION). Blood. 2014;123:494-500.

5. Hochhaus A, Masszi T, Giles FJ, et al. Treatment-free remission following frontline nilotinib in patients with chronic myeloid leukemia in chronic phase: results from the ENESTfreedom study. Leukemia. 2017;31:1525-31.

6. Wylie AA, Schoepfer J, Jahnke W, et al. The allosteric inhibitorABL001 enables dual targeting of BCR-ABL1. Nature. 2017;543:733-7.

7. Sopper S, MustjokiS, WhiteD, et al. Reduced CD62L expression on T cells and increased soluble CD62L levels predict molecular response to tyrosine kinase inhibitor therapy in early chronic-phase chronic myelogenous leukemia. JClin Oncol. 2017;35:175-84.

8. Pfirrmann M, Baccarani M, Saussele S, et al. Prognosis of long-term survival considering disease-specific death in patients with chronic myeloid leukemia. Leukemia. 2016;30:48-56.

9. Mahon FX, Richter J, Guilhot J, et al. Cessation of tyrosine kinase inhibitors treatment in chronic myeloid leukemia patients with deep molecular response: results of the EuroSki Trial. Blood. 2016;128(22):787.

10. Ilander M, Olsson-Strömberg U, Schlums H, et al. Increased proportion of mature NK cells is associated with successful imatinib discontinuation in chronic myeloid leukemia. Leukemia. 2017;31:1108-16.

11. ReaD, HenryG, KhaznadarZ, etal. Natural killer-cell counts are associated with molecular relapse-free survival after imatinib discontinuation in chronic myeloid leukemia: the IMMUNOSTIM study. Haematologica. 2017;102:1368-77.

12. Schütz C, Inselmann S, Sausslele S, et al. Expression of the CTLA-4 ligand CD86 on plasmacytoid dendritic cells (pDC) predicts risk of disease recurrence after treatment discontinuation in CML. Leukemia. 2017;31:829-36.

13. Gisslinger H, Zagrijtschuk O, Buxhofer-Ausch V, et al. Ropeginterferon alfa-2b, a novel IFN $\alpha$-2b, induces high response rates with low toxicity in patients with polycythemia vera. Blood. 2015;126:1762-9.

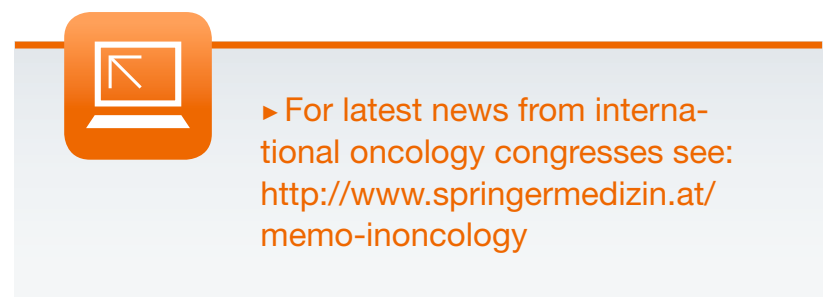

